\title{
Instability in a non-ohmic/ohmic fluid interface under a perpendicular electric field and unipolar injection
}

\author{
F. Vega and A. T. Pérez \\ Departamento de Electrónica y Electromagnetismo, Facultad de Física, Universidad de Sevilla, \\ Reina Mercedes s/n, 41012 Sevilla, Spain
}

(Received 6 January 2002; accepted 2 May 2002; published 1 July 2002)

\begin{abstract}
We set the equations for the linear electrohydrodynamic instability of an interface between two fluids, subjected to a perpendicular field and a unipolar charge injection. One of the fluids is modeled as being in non-ohmic regime (insulating), whereas the other is ohmic. A new interfacial instability mechanism is described, which may account for the Rose-window instability. The equations are analytically solved in the limit of long wavelength and neglecting the fluid motion. We show that this limit applies well to the case of an air-ohmic liquid interface. The applicability to a liquid-liquid interface is also analyzed. (C) 2002 American Institute of Physics.
\end{abstract}

[DOI: $10.1063 / 1.1488146]$

\section{INTRODUCTION}

Electrohydrodynamic (EHD) stability of fluids under a perpendicular electric field to the interface has been extensively studied in the case of no injection. ${ }^{1-3}$ But systems under charge injection, which allow a steady space charge distribution, show instabilities with some peculiarities that make their study interesting. The works by Mima et al. ${ }^{4}$ and Giannetta and Ikezi ${ }^{5}$ studied the dynamics of a helium liquid interface negatively charged. But they do not consider the existence of a space charge either in the liquid or in the vapor. The injection in a plane geometry was theoretically studied by Atten and Moreau in an insulating liquid layer. ${ }^{6}$ This study was later extended to double layer systems: air/ insulating liquid and insulating/perfect conducting liquids..$^{7-10}$ From this, the initial problem without space charge distribution in the no injection case turns with injection into a more complicated problem with $\boldsymbol{\nabla} \cdot \mathbf{E} \neq 0$.

In this work we study the EHD interfacial instability for a two fluid system: a non-ohmic (insulating) fluid layer is superposed to an ohmic fluid layer with an arbitrary conductivity. And we show that there is a new interfacial instability mechanism. Our study was initially motivated by some recent experiments ${ }^{11}$ in which an instability of a plane liquid surface is observed. This instability appears when a thin layer of low conducting liquid (in ohmic or non-ohmic regime) is subjected to a corona discharge in air. The instability has some peculiarities that distinguish it from previous known EHD phenomena. It appears in highly insulating liquids where the existence of space charge in the volume of the liquid is expected (silicon oil, for example), but also in more conducting liquids (corn or castor oil), where the conduction mechanism is expected to be ohmic and the space charge must be absent. Another conspicuous characteristic of this instability is that it gives rise to a pattern of polygonal cells with a size much greater than the liquid layer thickness. In other words, it is characterized by a critical long wavelength. The beautiful pattern that is obtained when the corona discharge is produced by a tip has led us to refer to this insta- bility as the Rose-window instability. The experimental results ${ }^{11,12}$ have induced us to think that the instability is essentially static, and due to the destabilizing effect of the electric pressure onto the liquid surface. This is the reason why the following analysis is essentially hydrostatics with the additional electric stresses at the surface.

After a considerable part of the study was accomplished we realized that, under certain circumstances, our conclusions were also of application to a liquid-liquid interface. Therefore we have chosen to present the problem in a more general scenario, involving two fluid layers. Although at the end we will neglect the fluid velocities, we think it is interesting to present and discuss the whole set of equations. In doing so, the reader will know what terms are neglected and the validity of the analysis will be better established. The applicability to a liquid-gas or a liquid-liquid interface is discussed throughout the paper.

The instability analysis is carried out in the standard way. The base stationary solution is perturbed in Fourier modes and a set of linear differential equations is obtained for the small perturbations of this base solution. The boundary conditions provide a set of algebraic equations for the coefficients of the solutions to the linear differential equations. The determinant corresponding to the coefficients of this system should be zero for a nontrivial solution to exist. This gives a function $U(k)$, where $U$ is the characteristic eigenvalue of the system and $k$ the wave number. In Sec. II we propose a general formulation of the problem of a nonohmic/ohmic interface subjected to injection. Section III discusses the solution for the unperturbed plane interface. Section IV is devoted to the formulation of the linear stability analysis. We formulate the problem in a general setting in order to show a set of equations whose solution would allow one to obtain the linear criterion in previous related works on instabilities in fluids under a perpendicular electric field, with or without injection. Nevertheless the general problem is not solved and we restrict to the solution in the limits where new instabilities are obtained. This is the case of an air-ohmic liquid interface, in Sec. V, where we make some simplifica- 


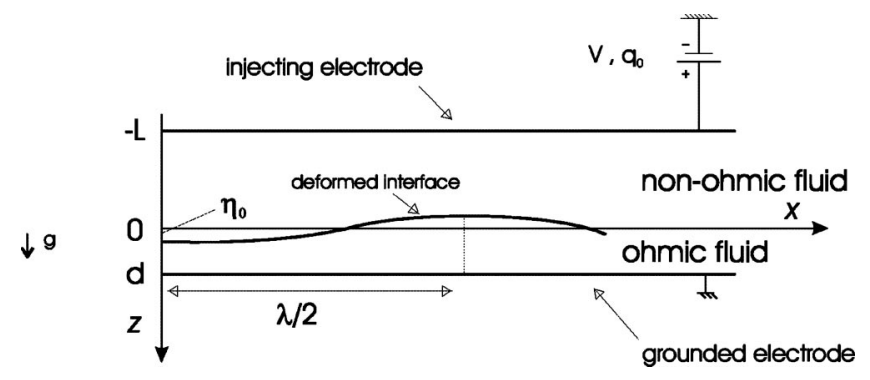

FIG. 1. Geometrical configuration of the system. The injecting electrode is at $z=-L$ and the collector electrode is at $z=d$. The interface is between both electrodes at $z=0$.

tions that allow one to obtain an analytical solution of the problem in the limit of long wavelength. Section VI is devoted to the liquid-liquid interface. And finally, in Sec. VII we present our conclusions and discuss possible generalizations of this work. In the appendices the detailed derivation and solution of the equations is presented. This is done in order to facilitate the reading and understanding of the physics involved.

\section{STATEMENT OF THE PROBLEM}

Let us consider two infinite parallel plane electrodes with a non-ohmic(insulating)/ohmic fluid interface in between. The electrodes are perfectly rigid metal plates. The non-ohmic fluid, which we assume to have a lower mass density, is over the ohmic fluid. The two fluids, initially at rest in an equilibrium state, are immiscible and incompressible. The interface is at $z=0$, being the non-ohmic layer at $z<0$ and the ohmic layer at $z>0$. The non-ohmic layer thickness is $L$ and the ohmic layer thickness is $d$. Figure 1 shows the geometrical configuration, where $h=L+d$ is the total length of the system. An applied voltage $V$ exists between both electrodes and, eventually, there is unipolar charge injection ${ }^{6}$ from the upper electrode. The charge is injected toward the lower electrode, reaching the ohmic fluid layer. Taking $h, V, \varepsilon_{i} V / h^{2}, K_{i} \varepsilon_{i} V^{2} / h^{3}, K_{i} V / h$, and $\varepsilon_{i} V^{2} / h^{2}$ as the units for distance, electric potential, space charge, current density, velocity and pressure, the set of differential equations in nondimensional form for the non-ohmic fluid layer is

$$
\begin{aligned}
& \mathbf{E}_{i}=-\boldsymbol{\nabla} \phi_{i}, \\
& \boldsymbol{\nabla} \cdot \mathbf{E}_{i}=q_{i}, \\
& \mathbf{j}_{i}=q_{i}\left(\mathbf{E}_{i}+\mathbf{v}_{i}\right), \\
& \frac{\partial q_{i}}{\partial t}+\boldsymbol{\nabla} \cdot \mathbf{j}_{i}=0, \\
& \boldsymbol{\nabla} \cdot \mathbf{v}_{i}=0, \\
& \frac{\rho_{r i}}{M^{2}}\left(\frac{\partial \mathbf{v}_{i}}{\partial t}+\left(\mathbf{v}_{i} \cdot \boldsymbol{\nabla}\right) \mathbf{v}_{i}\right)-\frac{1}{T} \nabla^{2} \mathbf{v}_{i}=\frac{\rho_{r i}}{U} \mathbf{e}_{z}-\boldsymbol{\nabla} p_{i}+q_{i} \mathbf{E}_{i} .
\end{aligned}
$$

We assume that the lower fluid layer is in ohmic regime and that the charge relaxation time is short enough in order to have $q_{c}=0$. Then, its governing equations are

$$
\begin{aligned}
& \mathbf{E}_{c}=-\boldsymbol{\nabla} \phi_{c}, \\
& \boldsymbol{\nabla} \cdot \mathbf{E}_{c}=0, \\
& \mathbf{j}_{c}=S \mathbf{E}_{c}, \\
& \boldsymbol{\nabla} \cdot \mathbf{v}_{c}=0, \\
& \frac{\rho_{r c}}{M^{2}}\left(\frac{\partial \mathbf{v}_{c}}{\partial t}+\left(\mathbf{v}_{c} \cdot \boldsymbol{\nabla}\right) \mathbf{v}_{c}\right)-\frac{\mu}{T} \nabla^{2} \mathbf{v}_{c}=\frac{\rho_{r c}}{U} \mathbf{e}_{z}-\boldsymbol{\nabla} p_{c} .
\end{aligned}
$$

The subscripts $i$ and $c$ stand for the non-ohmic (insulating) and ohmic (conducting) fluids, respectively. $\mathbf{E}$ is the electric field, $\phi$ is the electric potential, $\mathbf{j}$ is the current density, $K$ is the ion mobility, $\mathbf{v}$ the fluid velocity. The boundary conditions for two rigid electrodes are

$$
\begin{aligned}
& \phi_{i}(-L)=1, \quad q_{i}(-L)=C, \quad \mathbf{v}_{i}(-L)=0, \\
& \phi_{c}(d)=0, \quad \mathbf{v}_{c}(d)=0 .
\end{aligned}
$$

The electromechanical boundary conditions at the interface, defined by the equation $F(x, y, z, t)=0$, are ${ }^{13,14}$

$\mathbf{n} \cdot\left\langle\varepsilon_{r} \mathbf{E}\right\rangle=\sigma_{s}, \quad \mathbf{n} \times\langle\mathbf{E}\rangle=0$,

$\frac{\partial \sigma_{s}}{\partial t}+v_{n}(\boldsymbol{\nabla} \cdot \mathbf{n}) \sigma_{s}+\boldsymbol{\nabla}_{s} \cdot \mathbf{K}_{s}+\mathbf{n} \cdot\langle\mathbf{j}\rangle=0$,

$\frac{\partial F}{\partial t}+v_{n} \mathbf{n} \cdot \nabla F=0, \quad \mathbf{n} \cdot\langle\mathbf{v}\rangle=0, \quad \mathbf{n} \times\langle\mathbf{v}\rangle=0$,

$\mathbf{s}_{j} \cdot\left\langle\frac{\mu_{r}}{T}\left(\boldsymbol{\nabla} \mathbf{v}+\boldsymbol{\nabla} \mathbf{v}^{T}\right)\right\rangle \cdot \mathbf{n}+\sigma_{s} \mathbf{s}_{j} \cdot \mathbf{E}=0, \quad j=1,2$,

$\mathbf{n} \cdot\left\langle\frac{\mu_{r}}{T}\left(\boldsymbol{\nabla} \mathbf{v}+\boldsymbol{\nabla} \mathbf{v}^{T}\right)\right\rangle \cdot \mathbf{n}-\langle p\rangle-\left\langle\frac{1}{2} \varepsilon_{r} E^{2}\right\rangle+\left\langle\varepsilon_{r}(\mathbf{n} \cdot \mathbf{E})^{2}\right\rangle$

$$
=\frac{1}{U \text { Bo }} \nabla \cdot \mathbf{n} \text {. }
$$

Equations (17) and (18) are the tangent and normal balance conditions in the interface, respectively. These are the nondimensional parameters appearing in Eqs. (6)-(18):

$$
\begin{aligned}
& T=\frac{\varepsilon_{i} V}{K_{i} \mu_{i}}, \quad M=\frac{\sqrt{\varepsilon_{i} /\langle\rho\rangle}}{K_{i}}, \quad U=\frac{\varepsilon_{i} V^{2}}{\langle\rho\rangle g h^{3}}, \\
& \mathrm{Bo}=\frac{\langle\rho\rangle g h^{2}}{\gamma}, \\
& C=\frac{q_{0} h^{2}}{\varepsilon_{i} V}, \quad S=\frac{\sigma_{c} h^{2}}{K_{i} \varepsilon_{i} V} .
\end{aligned}
$$

It will be useful as well to define the number $P$ :

$$
P=\frac{\varepsilon_{i} \gamma h}{K_{i}^{2} \mu_{i}^{2}}
$$

and in (6) and (11):

$$
\rho_{r c}=\frac{\rho_{c}}{\langle\rho\rangle}, \quad \rho_{r i}=\frac{\rho_{i}}{\langle\rho\rangle}, \quad \mu=\frac{\mu_{c}}{\mu_{i}},
$$

while in the boundary conditions (14), (17), and (18) appear: 


$$
\varepsilon_{r}=\frac{\varepsilon_{l}}{\varepsilon_{i}}, \quad \mu_{r}=\frac{\mu_{l}}{\mu_{i}}
$$

where $l=i, c$ for the non-ohmic and the ohmic layer, respectively.

In the equations $\mathbf{n}$ refers to a vector normal to the surface (directed toward the lower fluid), $\mathbf{s}$ to a vector tangent to the surface, $\nabla_{s}$ is the operator $\boldsymbol{\nabla}-\mathbf{n}(\mathbf{n} \cdot \boldsymbol{\nabla}), \sigma_{s}$ is the surface charge density, $\mathbf{K}_{s}$ is the surface current density, $\gamma$ is the surface tension, $\rho$ the mass density, $g$ the acceleration of gravity, $\sigma_{c}$ is the ohmic fluid conductivity, and $\langle A\rangle=A^{+}$ $-A^{-}$indicates the jump of the magnitude $A$ at the interface ( $A^{+}$is the value of the magnitude inside the lower fluid). The parameter $T$ is the ratio of electrical and viscous forces in the non-ohmic layer and is the control parameter in the stability of a layer of insulating liquid subject to unipolar injection. ${ }^{6}$ The parameter $U$ is a relation between the electrical and gravitational forces acting on the interface and is the control parameter in the interfacial instability. The Bond number Bo is the ratio of the gravitational and capillary forces. The nondimensional conductivity $S$ plays an essential role in our study. It is a relation between the ohmic current density $\sigma_{c} V / h$ corresponding to the system with only the ohmic fluid and the non-ohmic current density $K_{i} \varepsilon_{i} V^{2} / h^{3}$ corresponding to the system with only the non-ohmic fluid. Two limit regimes are distinguished, depending on the value of $S$ : for example, for two layers of equal thickness, if $S \ll 1$ the voltage drop occurs mainly in the ohmic fluid, whereas if $S \gg 1$ the lower fluid behaves as a deformable electrode. The parameter $S$ can be considered as well as a relation between the ohmic and non-ohmic characteristic times $\varepsilon_{i} / \sigma_{c}$ and $h^{2} / K_{i} V$. Finally, the boundary condition for the space charge density $q(-L)=C$ is extremely important in unipolar injection problems and indicates that we have a space charge source at $z=-L$. The strength of the injection is characterized by the injection level parameter $C=q_{0} h^{2} /\left(\varepsilon_{i} V\right)$, where $q_{0}$ is the physical space charge density at the injecting electrode $z=-L$. In this work we will suppose that we can vary the electric potential and the space charge density independently (autonomous injector model ${ }^{6}$ ) and then the boundary conditions $\phi_{i}(-L)=1$ and $q(-L)=C$ are independent.

\section{ELECTRIC FIELD PERPENDICULAR TO THE INTERFACE AND SPACE CHARGE INJECTION: BASE STATE SOLUTION}

Both fluids are initially at rest $\mathbf{v}=\mathbf{0}$. In this state, $\mathbf{n}$ and the applied electric field are parallel to the acceleration of gravity $\left(\mathbf{n}=\mathbf{e}_{z}, \mathbf{E}=E \mathbf{e}_{z}\right)$, which implies that the electric field is perpendicular to the interface. Taking this into account we can greatly simplify the set of equations (1)-(18) that reduce to the system:

$$
\begin{aligned}
& j_{i}=q_{i} E_{i}, \quad \frac{d E_{i}}{d z}=q_{i}, \\
& j_{c}=S E_{c}, \quad \frac{d E_{c}}{d z}=0
\end{aligned}
$$

with the electrical boundary conditions from (12) to (15):

$$
\begin{aligned}
& \phi_{i}(-L)=1, \\
& q_{i}(-L)=C, \\
& \phi_{c}(d)=0, \\
& \phi_{c}(0)=\phi_{i}(0), \\
& j_{c}(0)=j_{i}(0)=j .
\end{aligned}
$$

The stationary electric field is

$$
\begin{aligned}
& E_{i}(z)=\sqrt{2 j(z+b)}, \quad z<0, \\
& E_{c}(z)=\frac{j}{S}, \quad z>0,
\end{aligned}
$$

where we need to find the two constants $j$, which is the current density, and $b$, which gives us $E\left(0^{-}\right)$. We can study the relation between $j$ and $b$ through Eqs. (26) and (27). From Eq. (27):

$$
b=\frac{j}{2 C^{2}}+L \text {. }
$$

Integrating Eqs. (31) and (32) from $z=-L$ to $z=d$ (electric potential equation) we have

$$
1=\sqrt{\frac{8}{9}} j\left[\left(\frac{j}{2 C^{2}}+L\right)^{3 / 2}-\left(\frac{j}{2 C^{2}}\right)^{3 / 2}\right]+\frac{j}{S} d .
$$

Clearly, from Eq. (34), the current density is a function of $C$. The expression for the nondimensional total surface charge (including the polarization charge) at the interface is

$$
\sigma_{s}=\frac{j}{S}-\sqrt{2 j b}
$$

If the parameter $S$ is small enough a positive surface charge tends to accumulate at the interface and in consequence the electric pressure pushes the lower fluid down. At a certain value $S^{\text {crit }}$ of $S$ the surface charge vanishes $\left(\sigma_{s}\right.$ $=0$ ). If we continue to increase $S$, the surface charge becomes negative and the electric pressure pulls up the ohmic fluid.

Figure 2(a) shows the current density and the electric field at the injector and at the interface plotted as a function of the injection parameter for $S \ll S^{\text {crit }}$. Figure $2(\mathrm{~b})$ is the corresponding plot for $S \gg S^{\text {crit }}$. In both cases there are two well-defined regions: a first region in the low $C$ values (weak injection) where the current density and $E_{i}(-L)$ are, respectively, increasing and decreasing functions of $C$, and $E_{i}(0)$ is decreasing for $S \ll S^{\text {crit }}$ but increasing for $S \gg S^{\text {crit }}$. A second region is rapidly reached, in which all magnitudes saturate and become constant. This is the space charge limited current regime (SCLC, see Ref. 13) and the system is said to be under strong injection.

In Fig. 3 we have plotted the current density $j$ in the case of low $S$ as a function of $C$ for different values of $d$. In this case, under strong injection, the current density decreases when the ohmic layer thickness increases. This "insulating" behavior tends to disappear if we switch off the space charge injection $(C \rightarrow 0)$. The critical $S^{\text {crit }}$ values versus $C$ are plotted in Fig. 4. 

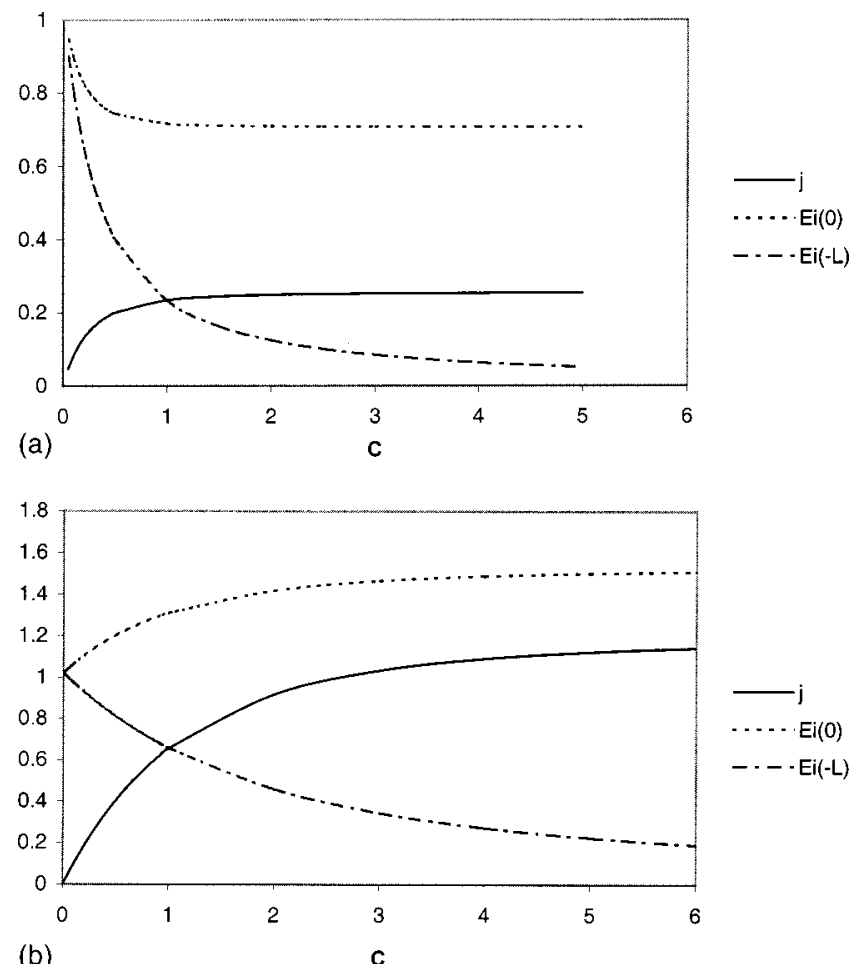

(b)

FIG. 2. (a) Reduced current density $j$, electric field at the injecting electrode $E_{i}(-L)$, and electric field at the interface $E_{i}(0)$ in a low $S$ regime, $S$ $=9.5 \times 10^{-3} \ll S^{\text {crit }}$, plotted vs the injection parameter $C$. When the system reaches to the space charge limited current regime (SCLC) all magnitudes become independent of the injection level $C$. All variables are nondimensional, except where indicated, as defined in Sec. III. (b) $j, E_{i}(-L)$, and $E_{i}(0)$ for a high $S$ regime, $S \gg S^{\text {crit }}$, plotted vs the injection parameter $C$. The weak injection region $(C \ll 1)$ and the SCLC regime are also observable in high $S$ regimes although with some peculiarities with respect to the low $S$ regime.

By analyzing the static electric pressure jump $\left\langle p_{e}\right\rangle$ $=\left\langle\varepsilon_{r} E^{2} / 2\right\rangle$ we can get some qualitative information on the interfacial instability mechanism in low and high $S$ regimes. We have plotted the electric pressure jump as a function of the layer thickness $d$ for a low $S$ value and it is always a decreasing function of the thickness (Fig. 5). This is a potentially unstable situation: given a perturbation of the surface there will be a higher electric pressure in the thinner areas and therefore the perturbation will be reinforced. As the tendency of the pressure keeps the same for all $d$ values there

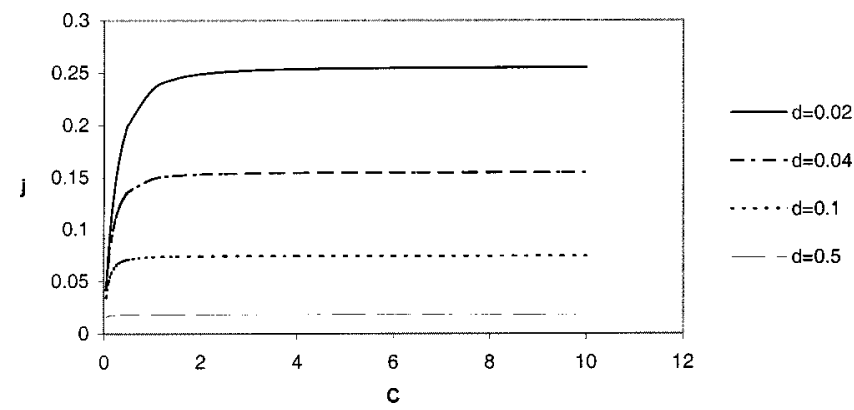

FIG. 3. Current density $j$ for $S=9.5 \times 10^{-3}$ as a function of the injection parameter $C$ for different values of the ohmic layer thickness $d$. The SCLC regime is more rapidly reached in thinner ohmic layers.

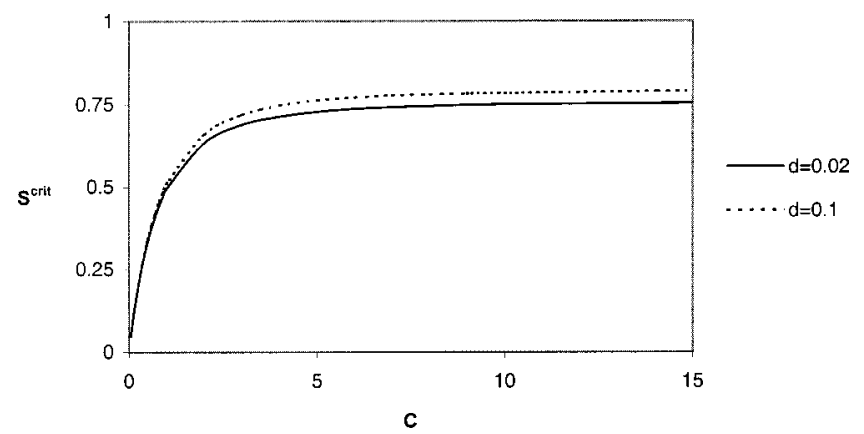

FIG. 4. Critical values $S^{\text {crit }}$ of the nondimensional conductivity (for which $\left.\sigma_{s}=0\right)$ as a function of the injection level $C$ for $d=0.02$ and $d=0.1$. $C$ $=10^{3}$.

should not be a limitation in the range of $d$ for the instability to occur. This is in contrast to the case of a perfect insulating lower fluid layer, where below certain value of $d$ the instability is no longer possible.,

The absolute value of the pressure jump for $S \gg S^{\text {crit }}$ (Fig. 6 ) is an increasing function of the ohmic layer thickness $d$. Again this is a potentially unstable situation, because for high $S$ regimes the pressure is directed upwards. We can get still another conclusion: since, under strong injection, low $S$ values allow a much higher surface charge accumulation than high $S$ values, instability should be expected to occur at lower applied potentials for low $S$ values. This is clear if it is compared with the magnitude of electric pressure jump in Figs. 5 and 6. Not only the sign of the pressure changes at a certain $S$, close to $S^{\text {crit }}$, but also the magnitude of the pressure is significantly different for $S \gg S^{\text {crit }}$ and $S \ll S^{\text {crit }}$. In any case a more precise determination of the critical values of the electric potential requires a linear instability analysis of the problem.

\section{LINEAR INSTABILITY EQUATIONS}

Let us study the evolution of a small perturbation of the steady solution. If the surface profile becomes irregular this will produce a deviation from the initial values of the electric field and the current density. We suppose that these perturbations are small enough to make possible a linear treatment. As in the static equations, all the magnitudes introduced here are nondimensional. The perturbed surface profile can be expressed:

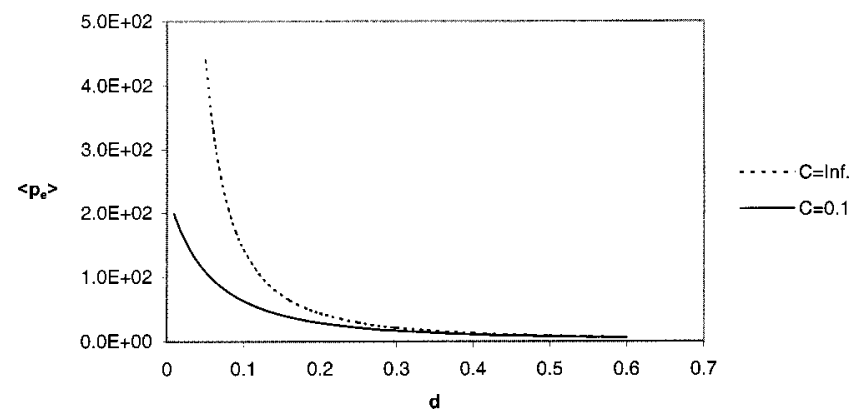

FIG. 5. Electric pressure jump $\left\langle(1 / 2) \varepsilon_{r} E^{2}\right\rangle$, for $S=9.5 \times 10^{-3}$, as a function of the ohmic layer thickness $d$ for different injection levels $C$. 


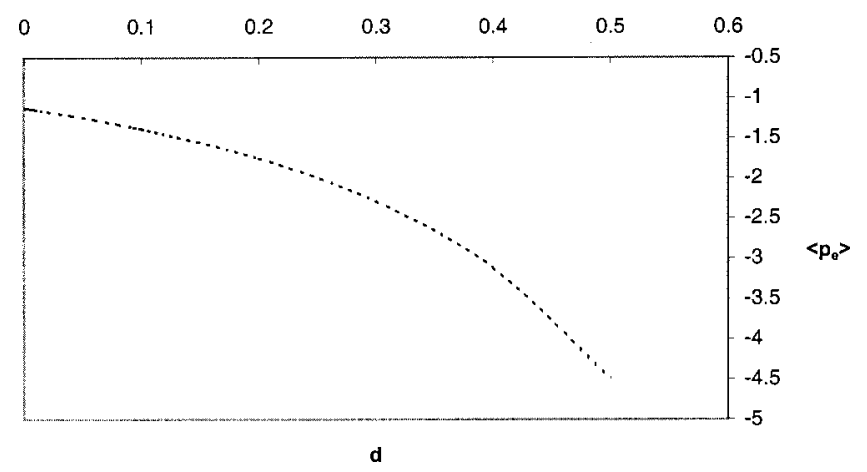

FIG. 6. Electric pressure jump in the case of a high $S$ regime plotted against thickness $d . C=10^{4}$.

$$
\eta(x, y, t)=\eta_{0} e^{\omega t+i\left(k_{x} x+k_{y} y\right)},
$$

where $\omega$ is a complex number, $k_{x}$ and $k_{y}$ are real, and $\eta_{0}$ is the deformation amplitude of the interface. The perturbations of all electric magnitudes can be expressed through the electric potential perturbation $\delta \phi$ that is of the form

$$
\delta \phi(x, y, z, t)=g(z) e^{\omega t+i\left(k_{x} x+k_{y} y\right)} .
$$

As a consequence of the surface deformation, a velocity perturbation term will appear. We only keep the $z$ component as velocity components tangent to the interface are related to the normal component through $\boldsymbol{\nabla} \cdot \delta \mathbf{v}=0$ :

$$
\delta v_{z}(x, y, z, t)=u(z) e^{\omega t+i\left(k_{x} x+k_{y} y\right)}
$$

being the normal velocity at the interface:

$$
u(0)=\omega \eta_{0} .
$$

Using Eqs. (1)-(11) a set of ordinary differential equations is deduced (see Appendix A):

$$
\begin{aligned}
& \left(\frac{d^{2}}{d z^{2}}-k^{2}\right)\left[\frac{T \rho_{r i}}{M^{2}} \omega-\left(\frac{d^{2}}{d z^{2}}-k^{2}\right)\right] u_{i} \\
& \quad=T k^{2} \frac{\sqrt{2 j}}{4}(z+b)^{-3 / 2}\left[g_{i}+4(z+b)^{2}\left(\frac{d^{2}}{d z^{2}}-k^{2}\right) g_{i}\right],
\end{aligned}
$$

$$
\begin{gathered}
\frac{4}{\sqrt{2 j}}(z+b)^{3 / 2} \omega\left(\frac{d^{2}}{d z^{2}}-k^{2}\right) g_{i}+4(z+b) \frac{d}{d z} \\
\times\left[(z+b)\left(\frac{d^{2}}{d z^{2}}-k^{2}\right) g_{i}\right]=\frac{d g_{i}}{d z}-u_{i},
\end{gathered}
$$

$\frac{d^{2} g_{c}}{d z^{2}}-k^{2} g_{c}=0$,

$\left(\frac{d^{2}}{d z^{2}}-k^{2}\right)\left[\frac{T \rho_{r c}}{M^{2}} \omega-\mu\left(\frac{d^{2}}{d z^{2}}-k^{2}\right)\right] u_{c}=0$.

The subscripts $i$ and $c$ refer to the non-ohmic and ohmic fluids, respectively. The general solution to this set of differential equations gives the perturbations of potential, velocity, and surface profile as a function of the wave number amongst other parameters. The solution to this system of equations will involve 13 unknown constants. The boundary conditions that follow from introducing a linear perturbation in (12)-(18) are

$g_{i}(-L)=0$,

$g_{i}^{\prime \prime}(-L)=0$,

$g_{c}(d)=0$

$g_{i}(0)-g_{c}(0)=(\sqrt{2 j b}-j / S) \eta_{0}$,

$$
\begin{aligned}
\sqrt{2 j} & {\left[b^{-1 / 2} g_{i}^{\prime}(0)+2\left(g_{i}^{\prime \prime}(0)-k^{2} g_{i}(0)\right) b^{1 / 2}\right] } \\
& =2 S g_{c}^{\prime}(0)+2 \sigma_{s} u_{i}^{\prime}(0)-2 \omega\left(g_{i}^{\prime}(0)-\varepsilon g_{c}^{\prime}(0)\right) .
\end{aligned}
$$

These are the electric boundary conditions. The boundary conditions (47) and (48) come from the continuity of electric potential and current density, respectively. From now on, the primes are always used to indicate the normal derivative $(d / d z)$. The boundary conditions for the velocity are

$$
\begin{aligned}
& u_{i}(-L)=u_{i}^{\prime}(-L)=0, \\
& u_{c}(d)=u_{c}^{\prime}(d)=0, \\
& u_{i}(0)=u_{c}(0)=\omega \eta_{0}, \\
& u_{i}^{\prime}(0)=u_{c}^{\prime}(0) .
\end{aligned}
$$

Equations (49) and (50) refer to the rigid electrode boundary conditions while (51) and (52) are the conditions due to the two fluids immiscibility (16). Finally the equilibrium of stresses tangent and normal to the interface are, respectively (see Appendix A),

$$
\begin{aligned}
& \frac{\mu}{T} u_{c}^{\prime \prime}(0)-\frac{1}{T} u_{i}^{\prime \prime}(0)+k^{2} \sigma_{s}\left(E_{c}(0) \eta_{0}-g_{c}(0)\right)=0 \\
& \frac{1}{M^{2}} \omega\left(\rho_{r i} u_{i}^{\prime}(0)-\rho_{r c} u_{c}^{\prime}(0)\right)+\frac{\mu}{T}\left(u_{c}^{\prime \prime \prime}(0)-3 k^{2} u_{c}^{\prime}(0)\right) \\
& \quad-\frac{1}{T}\left(u_{i}^{\prime \prime \prime}(0)-3 k^{2} u_{i}^{\prime}(0)\right)+k^{2} \sqrt{\frac{j}{2 b}} g_{i}(0) \\
& -k^{2}\left(\sqrt{2 j b} g_{i}^{\prime}(0)-\frac{\varepsilon j}{S} g_{c}^{\prime}(0)\right)+k^{2} \frac{1}{U}\left(\frac{k^{2}}{B o}+1\right) \eta_{0}=0 .
\end{aligned}
$$

Equations (44)-(54) set up a homogeneous system of equations for the constants of integration of Eqs. (40)-(43) and the deformation amplitude $\eta_{0}$. The determinant of the coefficients in this homogeneous system must be zero in order to have a possible nonzero solution for the perturbations. The zero determinant results in a dispersion relation $\omega(k)$, which is also a function of the nondimensional parameters that characterize the problem. This dispersion relation would account for all EHD instabilities in a non-ohmic/ohmic interface, including the cases without injection. ${ }^{1}$

If we assume that the principle of exchange of instabilities applies (for a definition of the principle of exchange of instabilities see Ref. 15) $\omega=0$ marks the point of incipient 
instability. This assumption can be justified in certain limits where the equations for $\omega=0$ are purely hydrostatic. For $\omega$ $=0$ the equations reduce to

$$
\begin{aligned}
& \left(\frac{d^{2}}{d z^{2}}-k^{2}\right)^{2} u_{i}=-T k^{2} \frac{\sqrt{2 j}}{4}(z+b)^{-3 / 2}\left[g_{i}+4(z+b)^{2}\right. \\
& \left.\times\left(\frac{d^{2}}{d z^{2}}-k^{2}\right) g_{i}\right] \\
& 4(z+b) \frac{d}{d z}\left[(z+b)\left(\frac{d^{2}}{d z^{2}}-k^{2}\right) g_{i}\right]=\frac{d g_{i}}{d z}-u_{i}, \\
& \frac{d^{2} g_{c}}{d z^{2}}-k^{2} g_{c}=0 \\
& \left(\frac{d^{2}}{d z^{2}}-k^{2}\right)^{2} u_{c}=0
\end{aligned}
$$

For $\omega=0$ all boundary conditions (44)-(53) remain unchanged except (48), (51) and (54). The condition (51) takes the following form:

$$
u_{i}(0)=u_{c}(0)=0
$$

and the condition (54):

$$
\begin{aligned}
& \frac{\mu}{T}\left(u_{c}^{\prime \prime \prime}(0)-3 k^{2} u_{c}^{\prime}(0)\right)-\frac{1}{T}\left(u_{i}^{\prime \prime \prime}(0)-3 k^{2} u_{i}^{\prime}(0)\right) \\
& +k^{2} \sqrt{\frac{j}{2 b}} g_{i}(0)-k^{2}\left(\sqrt{2 j b} g_{i}^{\prime}(0)-\frac{\varepsilon j}{S} g_{c}^{\prime}(0)\right) \\
& +k^{2} \frac{1}{U}\left(\frac{k^{2}}{B o}+1\right) \eta_{0}=0 .
\end{aligned}
$$

\section{THE CASE OF AN AIR-LIQUID INTERFACE IN THE LONG WAVELENGTH LIMIT}

Let us consider the case of an air-liquid interface. Since the ionic mobility in the air is high we can neglect the effect of fluid motion compared to ion transport by the electric field in the electric current density $\left(E_{i} \gg v_{i}\right) .{ }^{7}$ Under this assumption the electrical part of the problem is decoupled from the velocity perturbations in the non-ohmic layer. The space charge induces motion of the air, the so-called corona wind, but this motion does not perturb the space charge distribution. In addition the motion in the air is not transmitted to the liquid layer because the dynamic viscosity for the air is orders of magnitude smaller than for the liquid. The differential equations for the electric potential and the velocity perturbations in the ohmic layer (42) and (43) are not coupled and they can be solved separately. Finally for $k \ll 1$, Eq. (60) shows that the liquid velocity in the ohmic liquid becomes negligible (see also Ref. 10). It follows immediately that the stationary instability mode, at $\omega=0$, in an air-liquid interface is purely hydrostatic. The electrical part of the linear perturbation equations is

$$
\begin{aligned}
& (z+b)^{2} g_{i}^{\prime \prime \prime}(z)+(z+b) g_{i}^{\prime \prime}(z)-\left[k^{2}(z+b)^{2}+\frac{1}{4}\right] g_{i}^{\prime}(z) \\
& -k^{2}(z+b) g_{i}(z)=0,
\end{aligned}
$$

$$
k^{2} g_{c}(z)-g_{c}^{\prime \prime}(z)=0 .
$$

The solution to Eqs. (61) and (62) are, respectively,

$$
\begin{aligned}
g_{i}(z)= & A_{i} F_{1,2}\left[\frac{1}{2} ; \frac{3}{2}, \frac{3}{4} ; \frac{1}{4} k^{2}(z+b)^{2}\right] \\
& +B_{i}(z+b)^{1 / 2} F_{1,2}\left[\frac{3}{4} ; \frac{1}{2}, \frac{5}{4} ; \frac{1}{4} k^{2}(z+b)^{2}\right] \\
& +C_{i}(z+b)^{3 / 2} F_{1,2}\left[\frac{5}{4} ; \frac{3}{2}, \frac{7}{4} ; \frac{1}{4} k^{2}(z+b)^{2}\right], \\
g_{c}(z)= & A_{c} e^{k z}+B_{c} e^{-k z} .
\end{aligned}
$$

The set of boundary conditions gets reduced to the electric boundary conditions (44)-(48) and the mechanical boundary condition in the normal direction to the interface, that now takes the simplified form

$$
\begin{aligned}
& \sqrt{\frac{j}{2 b}} g_{i}(0)-\left(\sqrt{2 j b} g_{i}^{\prime}(0)-\frac{\varepsilon j}{S} g_{c}^{\prime}(0)\right) \\
& +\frac{1}{U}\left(\frac{k^{2}}{\mathrm{Bo}}+1\right) \eta_{0}=0 .
\end{aligned}
$$

The $F$ functions in (63) are called hypergeometric functions. ${ }^{16}$ In our problem they are of order $(1,2)$. The solutions of the differential equation (61) are combinations of the $F_{1,2}$ functions. They are power series of the following form:

$$
\begin{aligned}
F_{1,2}(a ; b, c ; z) & =\sum_{k=0}^{\infty} \frac{(a)_{k}}{(b)_{k}(c)_{k}} \frac{z^{k}}{k !} \\
& =1+\frac{a}{b c} z+\frac{a(a+1)}{b(b+1) c(c+1)} \frac{z^{2}}{2}+\cdots .
\end{aligned}
$$

In the air-ohmic liquid, interfaces other than the interfacial instability mechanism are nonpresent or negligible. EHD instabilities due to volume forces are absent in the ohmic fluid layer since $q_{c}=0$. And also convection in the air does not induce convection in the liquid due to the huge differences of the viscosity coefficients. Generally both mechanisms, convective and interfacial, ${ }^{9,10}$ are present and influence each other but from the above-mentioned considerations we may conclude that the instability in the case of an air/ohmic liquid interface is governed by the electric pressure acting on the surface.

\section{A. Relevant parameters}

As from (34) and (33) the current density $j$ and $b$ are functions of $C$, it is clear from Eqs. (63) to (65) that the only nondimensional numbers from (19) to (21) that remain in the problem are

$$
U=\frac{T^{2}}{P \mathrm{Bo}}=\frac{\varepsilon_{0} V^{2}}{\langle\rho\rangle g h^{3}}, \quad \text { Вo }=\frac{\langle\rho\rangle g h^{2}}{\gamma}
$$

and

$$
C=\frac{q_{0} h^{2}}{\varepsilon_{i} V}, \quad S=\frac{\sigma_{c} h^{2}}{K_{i} \varepsilon_{i} V} .
$$




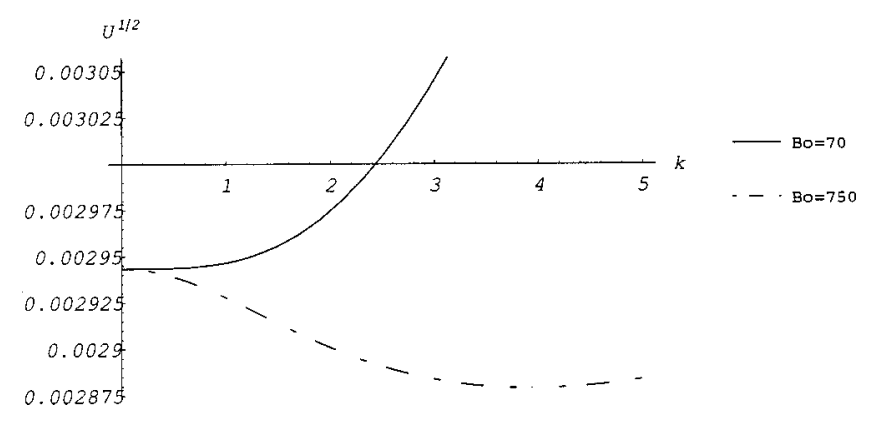

FIG. 7. Dispersion relation $U(k)$ with $S=9.5 \times 10^{-3}, d=0.02, C=10^{3}$. We observe the small influence of Bond number in the magnitude of $U_{c}$, even for two very different values of Bo.

Solving the determinant (see Appendix B) coming from the boundary conditions (44)-(48), and (65) we get the dispersion relation for an air/ohmic fluid interface at $\omega=0$. This relation yields the following function:

$$
U=U(k, \text { Bo, } C, S, \varepsilon, d) \text {. }
$$

Given a set of values for Bo, $C, S, \varepsilon, d$ the minimum of $U(k), U_{c}$, gives the instability criterion. For $U>U_{c}$ a few modes, different $k$, become unstable. We will denote as $k_{c}$ the value of $k$ which gives the minimum of $U(k)$. The Bond number $\mathrm{Bo}$ is the parameter controlling the critical wave number $k_{c}$ because in an interfacial instability the characteristic instability wavelength $k_{c}$ is given by the equilibrium between gravitational force and surface tension (see, e.g., Refs. 1 and 9). For $\mathrm{Bo} \rightarrow 0$ this characteristic wavelength tends to infinity and $U_{c}$ becomes independent of Bo. And conversely, the lower the surface tension (high Bo values) the lower the wavelength of the surface profile in the instability and the greater the influence of Bo in the critical voltage. Anyway, even for critical values $k_{c}>0$ the influence of surface tension on the critical voltage in the long wave region is not very important, remaining $U_{c}\left(k_{c}>0\right)$ of the same order as $U_{c}\left(k_{c}=0\right)$. In the following sections we show the critical values for an air/liquid interface analyzing separately the different ranges of the $S$ parameter.

\section{B. Low nondimensional conductivity ( $\left.S<S^{\text {crit }}\right)$}

For low $S$ values, the interface is charged with the same sign of the injecting electrode. The electric pressure pushes down the liquid layer in this case. Figure 7 is a plot of the function $U(k)$ for a value of $S$ smaller than that at which the interfacial charge changes sign and two different values of Bo. Above the line $U(k)$ the plane interface is unstable, below it is stable. Depending on the value of Bo the minimum is at $k=0$ or at some finite but small value $k>0$. However, below certain value of Bo the minimum is always at $k=0$ and the influence on the value of $U_{c}$ (the minimum value of $U$ at which instability is possible) is small.

Figure 8 shows the great influence that has the injection parameter $C$ in the weak injection region, as for low $C$ the $U_{c}$ values grow making it necessary to have very high critical voltages $V_{c}$ at the upper electrode to make possible the instability. As soon as we reach the strong injection region, $U_{c}$ becomes independent of $C$. This is a consequence of the

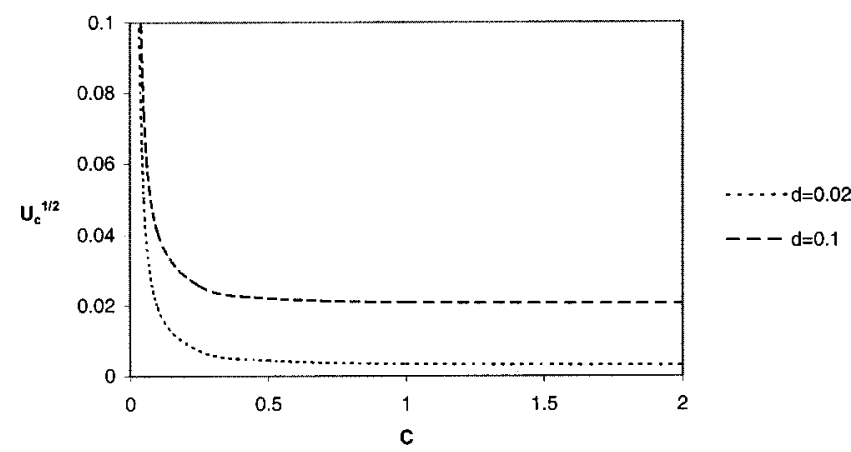

FIG. 8. A comparison of the typical minima of $U$ in the weak injection region $C \ll 1$ and the strong injection region $C \gg 1$. $S=9.5 \times 10^{-3}$, $\mathrm{Bo}=0.1$.

saturation of all the electric magnitudes in the SCLC regime. We can see in Fig. 8 the variation of $U_{c}$ as a function of the ohmic layer thickness $d$ in the strong injection regime. It is clear from the graph that thin ohmic layers become unstable at very low potentials under strong injection conditions. In the limit $S \rightarrow 0$ under strong injection the current density is $j=S / d$ and the dispersion relation (69) gives $U_{c}=d^{3} / \varepsilon$ if $\mathrm{Bo} \rightarrow 0$.

\section{Near critical nondimensional conductivity $\left(S \sim S^{\text {crit }}\right)$}

Figure 9(a) shows $U_{c}$ as a function of $S$ in the strong injection limit. As we can see in Fig. 9(a) there is a transition region where $U_{c}$ takes negative values, which means that the system is always stable. This region coincides with the range of $S$ where the surface density charge changes sign and marks the separation between the two different instability mechanisms in the low and high $S$ regimes that we anticipated in Sec. III. To the left of the intermediate region we have the instability mechanism for low $S$ regimes, which we think is responsible for the so-called Rose-window instability. And to the right we have the instability that corresponds to the well-known instability of a layer of conducting liquid subject to an electric field. As we see, the charge injection allows one to have instabilities at lower electric potentials in low $S$ regimes than in high $S$ regimes. If a charge injection was not applied to low $S$ regimes, the EHD interfacial instability would only be possible at higher electric potentials. The transition values $S^{\text {crit }}$ depend on the injection parameter $C$ as shown in Figs. 9(b) and 9(c). When $C$ tends to zero $S^{\text {crit }}=0$ and there is only the high $S$ regime mechanism. If we increase charge injection, $S^{\text {crit }}$ grows up to a limit value given by the saturation of the space charge limited current regime.

\section{High nondimensional conductivity ( $S>S^{\text {crit }}$ )}

Except for the values of $S$ close to the asymptote that separates the intermediate and the $S>S^{\text {crit }}$ regions, the variation of the eigenvalues with $C$ for $S>S^{\text {crit }}$ is not as dramatic as in the low $S$ regime region [Figs. 9(a)-9(c)], $U_{c}$ being of the same order in the cases of weak and strong injection (see Fig. 10). This is expected for $S>S^{\text {crit }}$ since in this case the 

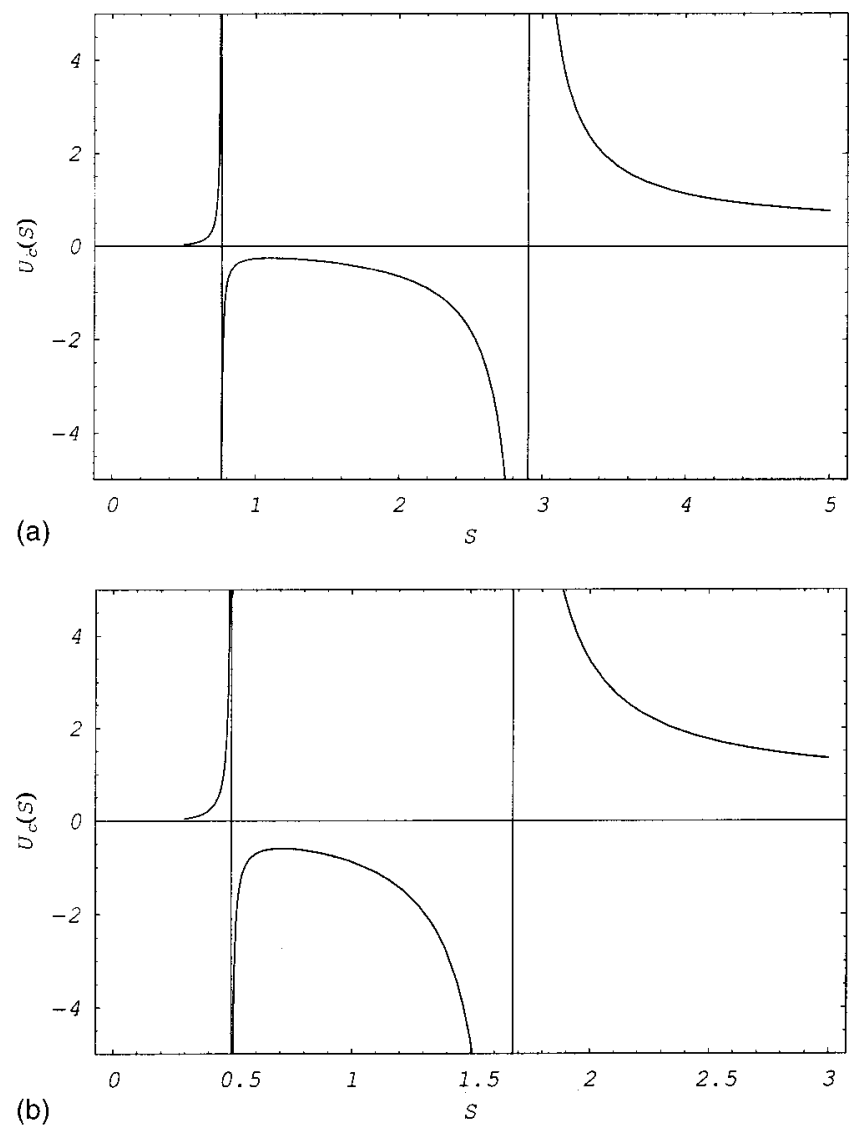

(b)

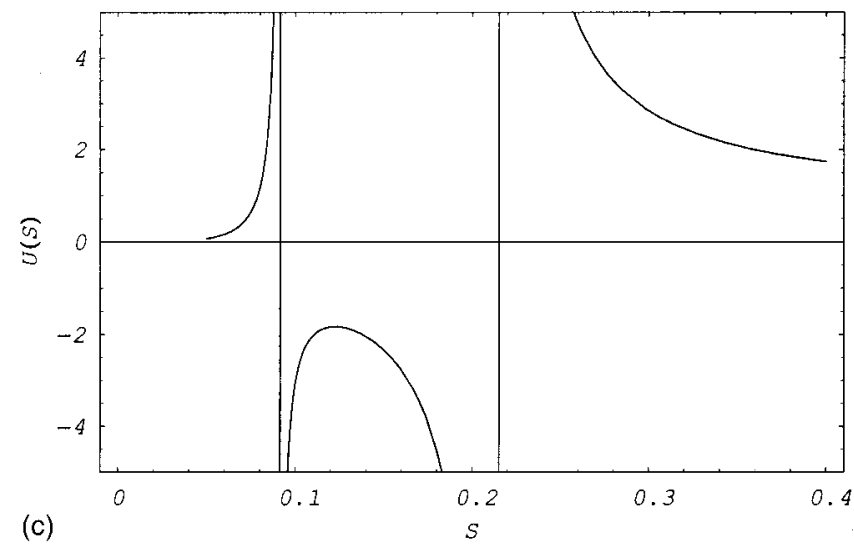

FIG. 9. (a) Critical parameter $U_{c}$ as a function of $S$ under strong injection $C=10^{4}$ and with an ohmic layer thickness $d=0.02, \mathrm{Bo}=0.1, k_{c}=0$. The difference in the order of magnitude of the critical values $U_{c}$ for low and high $S$ is remarkable, being much higher for high $S$. The instability is absent $(U<0)$ in the intermediate region, where the surface charge density is near zero. (b) The region of transition between low $S$ and high $S$ regime with an injection level $C=1$, being $d=0.02, \mathrm{Bo}=0.1, k_{c}=0$. (c) The transition between both regimes for weak injection $C=0.1$. As we see the transition region tends to zero for $C \rightarrow 0$. In the limit of no injection $C=0$ the high $S$ mechanism type occupies all the range of nondimensional conductivities and the instability is observable in low apparent conductivities only at very high potentials. $d=0.02, \mathrm{Bo}=0.1, k_{c}=0$.

interfacial instability mechanism is similar with and without injection, as in both cases the liquid is pulled toward the upper electrode by the electric pressure.

Clearly, the limit $S \rightarrow \infty$ corresponds to high conducting liquids. In Fig. 11 we present the typical critical curves for interfacial instabilities in perfect conducting liquids ${ }^{1,9}$ that

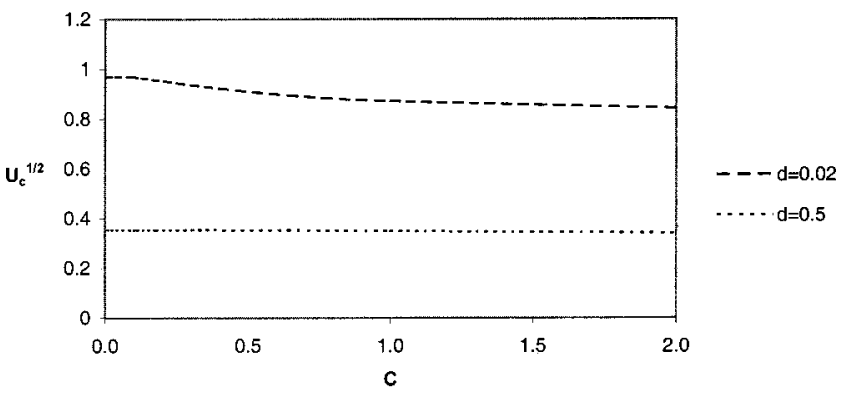

FIG. 10. Variation with the injection parameter $C$ of the critical $U_{c}$ for a high conducting liquid. Thickness $d=0.02,0.5$. If we compare these two curves we see that the behavior of $U_{c}$ with $d$ is the opposite to that for a low $S$ regime. $\mathrm{Bo}=0.1, k_{c}=0$.

we have obtained for different Bo values and $d=0.02$. Even for strong charge injection, they are very similar to those obtained by other authors in former studies without injection. This confirms that the instability mechanism, for small $d$, in high conducting liquids is the same with and without charge injection.

The well-known instability of a layer of a perfect conductor without injection is governed by the relation, in dimensional magnitudes, ${ }^{2}$

$$
-\langle\rho\rangle g-\gamma k^{2}+\varepsilon_{0} k E^{2} \operatorname{coth}(k L)=0 .
$$

For $k=0$ the instability criterion is

$$
\varepsilon_{0} E^{2}=\langle\rho\rangle g L,
$$

which corresponds to $U=L^{3}$ in nondimensional magnitudes. We have correctly obtained this relation from (69) for $C$ $=0$. The corresponding limit for $C \rightarrow \infty$, Bo $\rightarrow 0$ gives $U^{1 / 2}$ $=(2 / 3) L^{3 / 2}$ and reproduces the result in Ref. 10 .

The main peculiarity of the case $C \rightarrow \infty$ with respect to the case $C=0$ in the region $S>S^{\text {crit }}$ comes from the existence of the asymptotes of the function $U_{c}(S)$ if there is injection [see Fig. 9(a)]. This will be clearer in a dimensional representation, as explained in Sec. VE.

\section{E. Comparison between theoretical and experimental critical values for low conducting liquids: Rose-window instability}

This work began as an attempt to theoretically describe long wave instabilities observed in low conducting and per-

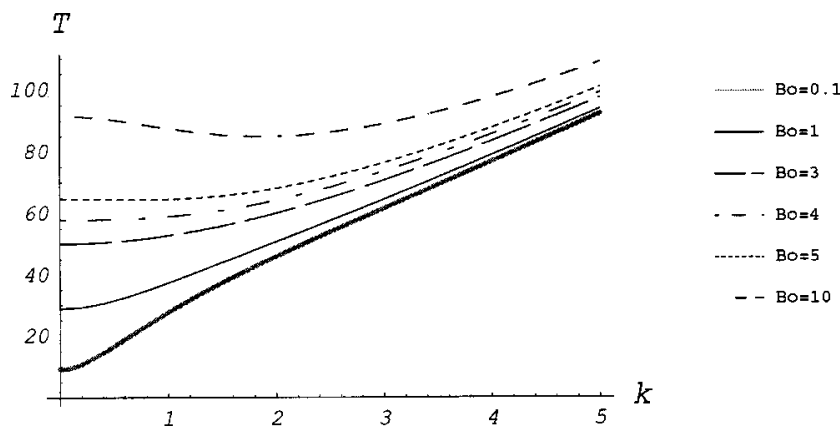

FIG. 11. Typical dispersion curves for high conducting liquids. The shape is similar to that found in no injection problems even for strong injection. $P$ $=2 \times 10^{3}, d=0.02, C=10^{4}$. 


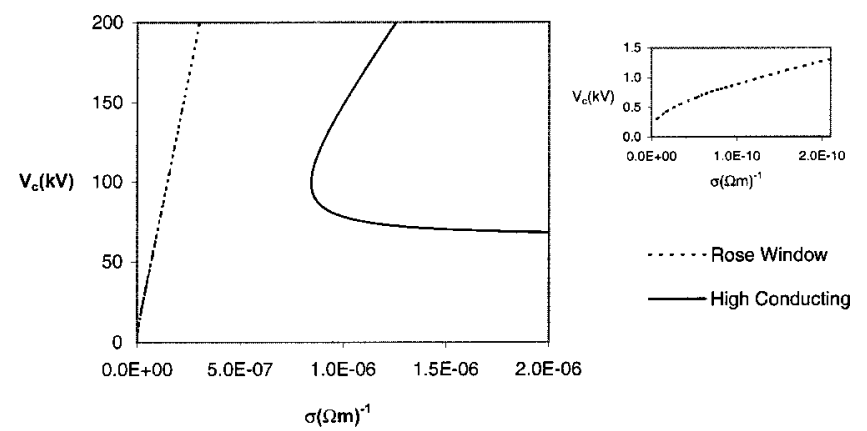

FIG. 12. Interfacial instability criterion vs conductivity. The curve at the left corresponds to the "down" pressure while the two branches at the right correspond to the "up" pressure. Nondimensional magnitudes: $\mathrm{Bo}=0.1$, $k_{c}=0, \quad d=0.02, \quad \varepsilon=4.69, \quad C=10^{3}$. Dimensional magnitudes: $h$ $=\sim 10^{-2} \mathrm{~m}, \quad K_{i} \sim 10^{-4} \mathrm{~m}^{2} /(\mathrm{V} \mathrm{s}), \quad \varepsilon_{i} \sim 10^{-11} \mathrm{C}^{2} N^{-1} \mathrm{~m}^{-2}, \quad\langle\rho\rangle g$ $\sim 10^{4} \mathrm{~N} \mathrm{~m}^{-3}$.

fect insulating liquids. ${ }^{11,8}$ This type of instability is the socalled Rose-window instability, and it is characterized by a much longer wavelength than the well-known classical EHD convective instability in insulating liquids. In order to predict the critical values for instability we have plotted in Fig. 12 the dimensional counterpart of Fig. 9(a). The curves indicate the critical voltages as a function of the liquid conductivity for certain values of the other magnitudes (see the caption of Fig. 12). Two curves are visible. The one on the left-hand side corresponds to the interfacial instability in low conducting liquids under strong injection. This is a new interfacial instability branch. This critical branch only appears with charge injection and we think it corresponds to the Rosewindow instability. The area delimited by this curve and the $\sigma=0$ axis is the low conducting unstable region. To the right-hand side, for more conducting liquids, there is another curve with two solutions for each conductivity value. The lower one marks the point of instability and for $\sigma \rightarrow \infty$ tends to $2 / 3$ of the classical value given by (70). Above the upper solution the plane surface is linearly stable. This upper solution, which is due to the existence of the asymptote in Fig. 9(a), is again new and appears only under charge injection. The area delimited by these two solutions is the high conducting unstable region. The intermediate area between the two critical curves is the stable region.

Some experimental values were obtained in our laboratory for a liquid layer subjected to ion injection from a corona discharge in air. The liquid that we used was castor oil, with a conductivity $\sigma_{c} \sim 10^{-10}(\Omega \mathrm{m})^{-1}$. We used corona discharge in a triode configuration. The triode configuration (Fig. 13) consists in a tip-grid-plane three electrode system that is intended to correct the nonuniform corona discharge distribution. The corona discharge is produced on the tip electrode that is above an intermediate metallic grid. The grid is parallel to the plane electrode containing the liquid layer. We apply a voltage $V_{g}$ to the grid that allows one to fix a perpendicular electric field over the liquid surface and lets a certain fraction of the ions coming from the tip pass toward the liquid. The experimental critical values of $V_{g}$ for the instability threshold range from 1.1 to $2.0 \mathrm{kV}$ for $d$ values (liquid layer thickness) from 1.2 to $2 \mathrm{~mm}$ (Fig. 14). These

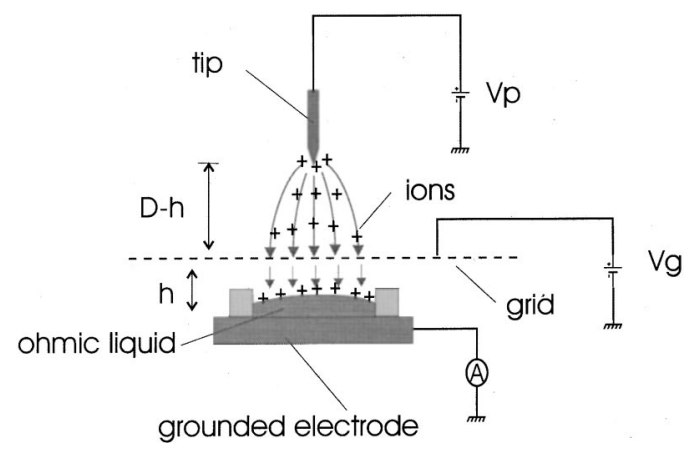

FIG. 13. Experimental setup: Triode configuration (three electrode system: tip-grid-plane). The tip is injecting ions that pass through the grid. We apply an electric potential $V_{g}$ at the grid while the plane (circular shape with $4 \mathrm{~cm}$ diameter) containing the liquid is grounded.

values are to be compared with the theoretical calculations, that go from 0.9 to $1.7 \mathrm{kV}$ (Fig. 12). An agreement is expected only in the order of magnitude since the systems are not exactly the same in the experimental and theoretical cases: in our experimental setup the grid is not a perfect rigid metal plate and the triode does not have the same correlation between voltage and current density than in the theoretical system. Logically, in these conditions complete agreement between experimental and theoretical values is not expected. In experimental setups like the tip-plane configuration (without a grid) the disagreement with the theory should be more important due to the effects of the nonhomogeneity of the electric field. We recall that, in such a low conducting liquid in contact with air, it is not possible to find perpendicular-field EHD instabilities at so low electric potentials if it is not by applying a charge injection. ${ }^{3}$

\section{LIQUID-LIQUID INTERFACE}

In studies on EHD instabilities limited to problems without space charge, ${ }^{3}$ the zero shear stress dynamics appears in two limits: perfect conducting (zero tangent electric field) and perfect insulating interfaces (zero surface free charge).

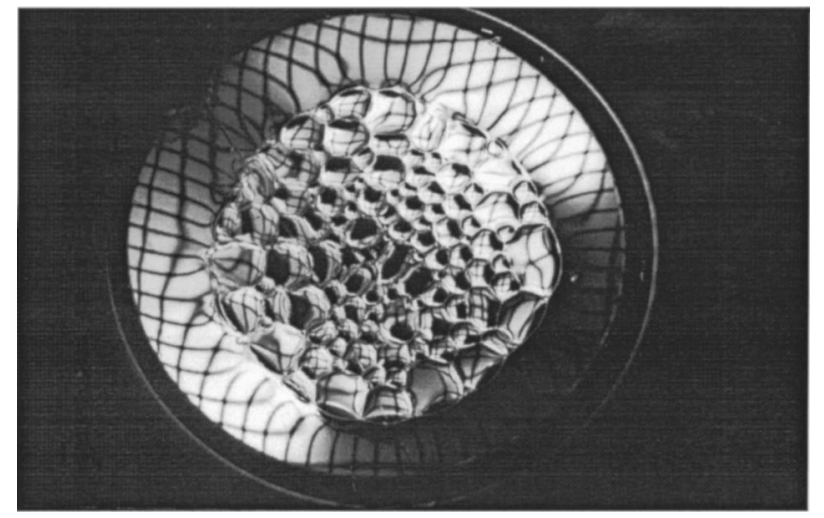

FIG. 14. Rose-window instability in a low conducting liquid (castor oil). $V p=12 \mathrm{kV}, V g=3.25 \mathrm{kV}, I=1.55 \mu \mathrm{A}, \sigma_{c} \sim 10^{-10}(\Omega \mathrm{m})^{-1}$. Tip-plane distance: $D=3.1 \mathrm{~cm}$. Grid-plane distance: $h=1.5 \mathrm{~cm}$. Liquid layer thickness: $d=1.2 \mathrm{~mm}$. Electrode area: $A=1.256 \times 10^{-3} \mathrm{~m}^{2}$. Liquid mass density: $\rho_{c}=958 \mathrm{~kg} / \mathrm{m}^{3} . \varepsilon=4.69$. Viscosity: $\mu_{c}=6 \times 10^{-4} \mathrm{~m}^{2} / \mathrm{s}$. Ion mobility: $K_{c}=4 \times 10^{-11} \mathrm{~m}^{2} /(\mathrm{V} \mathrm{s})$. 
These two limiting cases are valid as well in the analogous problems with space charge injection. With or without injection, in the long wave region $k \rightarrow 0$ the shear stress will be zero whatever the electric properties of the two fluids involved in the problem [see Eqs. (A12) and (A13)]. In these cases all the boundary conditions for the linear velocity perturbations are then homogeneous at $\omega=0$. If besides the differential equations of the velocity and electric potential linear perturbations are decoupled the interfacial instability mechanism will be purely static and then the solution of the electrical part itself gives the correct criterion at $\omega=0$. When are the electric potential and velocity differential equations decoupled? For sure, in $C=0$ problems. ${ }^{1,3}$ And as well in an injection problem $(C \neq 0)$ if the ion mobility of the nonohmic fluid is high enough. This is the case for an air layer over an ohmic liquid. However for a two liquid non-ohmic/ ohmic interface there is a strong coupling between the electric and viscous terms and convective instabilities may first appear. Atten and Koulova-Nenova have studied the transition from interfacial to convective instabilities in injection problems in the particular case of an insulating/perfect conducting interface in Refs. 9 and 10. In those works it is demonstrated that if $P=T^{2} /(U \mathrm{Bo})$ is low enough the interfacial instability will occur before the convective one even for strong injection (note that the special case $C=0$ gives the criterion by Taylor ${ }^{1}$ ). Similar curves should be expected in a non-ohmic/ohmic interface with an arbitrary conductivity. We expect that the instability criteria we have obtained in the present work are applicable in the long wave limit (low Bo) even for a two liquid interface if $P$ is small enough. (Nevertheless, for a more precise analogy it should be taken into account that the unit for distance in Ref. 9 is not the total length $h$ but the insulating liquid layer thickness $L$.) In fact we can easily see that Eqs. (55) and (58) in the limit $k \rightarrow 0$ reduce to

$$
\begin{aligned}
& \frac{d^{4} u_{i}}{d z^{2}}=0, \\
& \frac{d^{4} u_{c}}{d z^{2}}=0 .
\end{aligned}
$$

In the long wave limit the differential equations of the velocity perturbations are not coupled to the electric part, that with the boundary conditions for $\omega=0$ leads to the trivial solution:

$$
u_{i}(z)=u_{c}(z)=0 .
$$

Then it is justified that the solution of the electrical part of the perturbation equations is the only one that we need to study the interfacial instability, although it is obvious that within this limit we are not able to reproduce convective instabilities, ${ }^{9}$ unless we solve completely the equations that we have set: differential equations (40)-(43) with boundary conditions (44)-(54).

To simplify, we have just put together the arguments in Refs. 3, 7, and 10 and we have applied them to a perpendicular-field non-ohmic/ohmic interface with an arbitrary conductivity, under unipolar injection. On the other hand, if the Bond number is not low enough and $k=0$ does not give the instability criterion, the values here obtained should reproduce the orders of magnitude of the instability thresholds as the capillary forces involved will not have a strong influence on the magnitude of the critical voltage value.

\section{DISCUSSION AND CONCLUSION}

In this paper we have obtained the linear instability criterion for double fluid layer system under a dc perpendicular electric field and unipolar injection. In classical studies such as those by Taylor and McEwan and Melcher, ${ }^{1-3}$ the sign of the surface free charge was determined by the ratio between the conductivities of the two fluids. In nondimensional form,

$$
\sigma_{s}=\left(\frac{\sigma_{u} / \sigma_{l}}{\epsilon_{u} / \epsilon_{l}}-1\right) E_{u},
$$

where $l$ refers to the lower fluid and $u$ to the upper one. Depending on the ratio of conductivities the electric pressure could push down or pull up the liquid surface but, from the electrostatic point of view, the instability mechanism was essentially the same.

The unipolar injection from one of the electrodes builds a surface charge on the interface given, in nondimensional form, by

$$
\sigma_{s}=\frac{j}{S}-\sqrt{2 j b} .
$$

The sign of the charge depends now, not only on the conductivity of the ohmic liquid, but also on the level of injection and the thicknesses of the layers. There is a certain value of $S$, the nondimensional conductivity, for which the surface charge is zero. Below and above this value the surface charge is of the sign of the injecting electrode or the opposite. On the contrary to the case without injection, the interfacial instability mechanism is different for each situation. In fact, the low $S$ value regime is characterized by much lower values of the critical voltage.

It is interesting to note that in the strong injection case and for $L \rightarrow 0$ (very small non-ohmic layer) the critical nondimensional conductivity tends to infinity (please note that $S^{\text {crit }}$ in Fig. 4 increases with $d=1-L$ ). This makes possible the low $S$ mechanism of instability even for high conducting liquids (which is not taken into account in the work by Taylor $\left.{ }^{1}\right)$. Although our study is only linear and does not apply to a finitely deformed interface, we can envisage, for these reasons and for those explained in the discussion of Fig. 12, that the injection from air into a conducting surface can play a stabilizing role. It is clear that we are describing a new interfacial instability mechanism that is not taken into account in previous related works on fluid interfaces under stationary perpendicular electric fields, ${ }^{1-3}$ and this is due to the introduction in the present work of the additional parameter $C$. Experimental techniques to produce electric fields with space charge distributions are commonly used in laboratories. Then new experimental evidence, unexplained by previous theoretical works, could appear. We think that an example of this could be the Rose-window instability. ${ }^{11}$ 
In conclusion we have undertaken a linear instability analysis of a perpendicular-field non-ohmic/ohmic fluid interface. In the case of an air-liquid interface we have obtained a purely hydrostatic system of equations. We have seen that the relevant parameter in the interfacial instability is the ratio between electric and gravitational pressures as the characteristic eigenvalue is $U=\varepsilon_{i} V^{2} /\left(\langle\rho\rangle g h^{3}\right)$. The Bond number $\mathrm{Bo}=\langle\rho\rangle g h^{2} / \gamma$ is the parameter that controls the change of the critical $k$ value: for low enough Bo values we always get the pure interfacial instability at $k_{c}=0$, and in this limit the criterion is exact. We have obtained in this limit the criterion for a perfect conducting free surface in agreement with former works on EHD instabilities without charge injection. From an analysis of the static solution we have seen the difference between the interfacial instability mechanism with and without charge injection. From this difference many particularities of the interfacial instabilities in low conducting liquids rise: charge injection makes possible the appearance of instabilities in these liquids at much lower critical voltages than in high conducting liquids. A semiquantitative comparison with existing experimental data has been made. We have shown that the analysis can be applied to a liquid/ liquid interface if the numbers Bo and $P$ are low enough. Finally we recall that all the solutions obtained are analytical and confirmed by experimental observations.

\section{ACKNOWLEDGMENTS}

The authors wish to thank Dr. P. Atten and Professor A. Castellanos for fruitful discussion. This work has been carried out with financial support from the Spanish Ministerio de Ciencia y Tecnología (MCYT) under Research Project No. BFM2000-1056.

\section{APPENDIX A: LINEAR PERTURBATION EQUATIONS}

\section{Differential equations}

Differential equation (40) is deduced by applying twice the rotational in the $z$ component of the corresponding equation for the linear perturbation in (6). Then we have

$$
\begin{array}{r}
-\left(\frac{d^{2}}{d z^{2}}-k^{2}\right)\left[\frac{T}{M^{2}} \omega-\left(\frac{d^{2}}{d z^{2}}-k^{2}\right)\right] u_{i} \\
=T k^{2}\left[\phi^{\prime \prime \prime} g_{i}-\phi^{\prime}\left(\frac{d^{2}}{d z^{2}}-k^{2}\right) g_{i}\right] .
\end{array}
$$

The second differential equation for the non-ohmic liquid comes from the charge conservation equation. The linear equation for the perturbation is the following:

$$
\begin{aligned}
& \omega\left(\frac{d^{2}}{d z^{2}}-k^{2}\right) g_{i}+\phi^{\prime \prime \prime} u_{i} \\
& \quad=\phi^{\prime \prime \prime} g_{i}^{\prime}+2 \phi^{\prime \prime}\left(\frac{d^{2}}{d z^{2}}-k^{2}\right) g_{i}+\phi^{\prime} \frac{d}{d z}\left(\frac{d^{2}}{d z^{2}}-k^{2}\right) g_{i} .
\end{aligned}
$$

In Eqs. (A1) and (A2) $-\phi^{\prime}$ stands for the static electric field, whose value is given by (31) and (32). Knowing that

$$
\frac{\phi^{\prime \prime}}{\phi^{\prime \prime \prime}}=-2(z+b), \quad \frac{\phi^{\prime}}{\phi^{\prime \prime \prime}}=-4(z+b)^{2}
$$

we get the equations in (40) and (41). The equations corresponding to the ohmic liquid (42) and (43) are easily deduced from the charge and momentum conservation equations considering that $q_{c}, \delta q_{c}=0$.

\section{Boundary conditions}

The electric potential continuity condition at the interface gives

$$
\Phi_{c}(\eta)=\Phi_{i}(\eta),
$$

where $\Phi$ stands for the electric potential solution for the perturbed system:

$$
\Phi=\phi+\delta \phi,
$$

$\delta \phi=g(z) e^{i\left(k_{x} x+k_{y} y\right)}$ being the electric potential perturbation. If the perturbation is small we can consider only the linear terms in the perturbation:

$\phi_{c}(0)+\frac{\partial \phi_{c}(0)}{\partial z} \eta+\delta \phi_{c}(0)=\phi_{i}(0)+\frac{\partial \phi_{i}(0)}{\partial z} \eta+\delta \phi_{i}(0)$,

which together with the continuity of static potential at $z$ $=0$ gives

$$
\delta \phi_{i}-\delta \phi_{c}-\left(E_{i}(0)-E_{c}(0)\right) \eta=0
$$

and with $E_{c}(0)=j / S$ and $E_{i}(0)=\sqrt{2 j b}$ we have

$$
g_{i}(0)-g_{c}(0)=\left(\sqrt{2 j b}-\frac{j}{S}\right) \eta_{0},
$$

which is Eq. (47). In a similar way we obtain the normal current density continuity condition:

$$
\mathbf{j}_{c}(\eta) \cdot\left(\mathbf{e}_{z}+\delta \mathbf{n}\right)=\mathbf{j}_{i}(\eta) \cdot\left(\mathbf{e}_{z}+\delta \mathbf{n}\right) .
$$

At first order $\mathbf{n} \cdot \delta \mathbf{n}=0$, then

$$
E_{i, z}(0) \delta q(0)+q(0) \delta E_{i, z}(0)=S \delta E_{c, z}(0),
$$

which leads to Eq. (48). The rest of boundary conditions are deduced in a similar way. Special attention may be given to the tangent and normal stress balances (17) and (18). Knowing that

$$
\delta \mathbf{n}=-\left(\frac{\partial}{\partial x} \mathbf{e}_{x}+\frac{\partial}{\partial y} \mathbf{e}_{y}\right) \eta, \quad \delta \mathbf{s}_{1}=\frac{\partial \eta}{\partial x} \mathbf{e}_{z}, \quad \delta \mathbf{s}_{2}=\frac{\partial \eta}{\partial y} \mathbf{e}_{z},
$$

the two components of the linear perturbation of the shear stress balance are

$$
\begin{gathered}
\left\langle\frac{\mu_{r}}{T}\left(\frac{\partial \delta v_{x}}{\partial z}+\frac{\partial \delta v_{z}}{\partial x}\right)\right\rangle+\sigma_{s}\left(E_{c}(0) \frac{\partial \eta}{\partial x}-\frac{\partial \delta \phi_{c}(0)}{\partial x}\right)=0, \\
\left\langle\frac{\mu_{r}}{T}\left(\frac{\partial \delta v_{y}}{\partial z}+\frac{\partial \delta v_{z}}{\partial y}\right)\right\rangle+\sigma_{s}\left(E_{c}(0) \frac{\partial \eta}{\partial y}-\frac{\partial \delta \phi_{c}(0)}{\partial y}\right)=0,
\end{gathered}
$$

applying $\partial / \partial x$ and $\partial / \partial y$, respectively, and adding these two components: 
$\left\langle\frac{\mu_{r}}{T}\left(\Delta_{s} \delta v_{z}-\frac{\partial^{2} \delta v_{z}}{\partial z^{2}}\right)\right\rangle+\sigma_{s}\left(E_{c}(0) \Delta_{s} \eta-\Delta_{s} \delta \phi_{c}(0)\right)=0$.

The operator $\Delta_{s}=\partial^{2} / \partial x^{2}+\partial^{2} / \partial y^{2}$ applied to the linear perturbations is $-k^{2}$. In consequence, the condition gets

$$
-\frac{1}{T}\left\langle\mu_{r}\left(k^{2} u+\frac{\partial^{2} u}{\partial z^{2}}\right)\right\rangle-k^{2} \sigma_{s}\left(E_{c}(0) \eta_{0}-g_{c}(0)\right)=0 .
$$

And as $\langle u\rangle=0$, the equation takes the form in (53). The linear perturbation of the normal stress balance is

$$
2\left\langle\frac{\mu_{r}}{T}\left(\frac{\partial \delta v_{z}}{\partial z}\right)\right\rangle-\frac{1}{U} \eta-\langle\delta p\rangle+\left\langle\varepsilon E \delta \phi^{\prime}\right\rangle=\frac{1}{U B_{0}} \nabla \cdot \mathbf{n},
$$

$\langle\delta p\rangle$ being the linear perturbation in the total pressure jump $\left\langle p^{t}\right\rangle=\langle p\rangle+\langle\delta p\rangle$. We can eliminate $\langle\delta p\rangle$ with the $x$ and $y$ components of the Navier-Stokes equations. These equations for the non-ohmic layer are

$$
\begin{aligned}
& \frac{\rho_{r i}}{M^{2}} \frac{\partial \delta v_{i x}}{\partial t}=-\frac{\partial \delta p}{\partial x}+\frac{1}{T} \nabla^{2} \delta v_{i x}-q \frac{\partial \delta \phi_{i}}{\partial x}, \\
& \frac{\rho_{r i}}{M^{2}} \frac{\partial \delta v_{i y}}{\partial t}=-\frac{\partial \delta p}{\partial y}+\frac{1}{T} \nabla^{2} \delta v_{i y}-q \frac{\partial \delta \phi_{i}}{\partial y} .
\end{aligned}
$$

Applying $\partial / \partial x$ and $\partial / \partial y$ to (A17) and (A18), respectively, and adding both of them we get

$$
\frac{\rho_{r i}}{M^{2}} \frac{\partial \delta v_{i z}}{\partial z \partial t}-\Delta_{s} \delta p-\frac{1}{T}\left(\nabla^{2} \frac{\partial \delta v_{i z}}{\partial z}+q \Delta_{s} \delta \phi_{i}\right)=0
$$

as $\boldsymbol{\nabla} \cdot \delta \mathbf{v}=0$. With this and $\boldsymbol{\nabla} \mathbf{n}=-\Delta_{s} \eta$ the linear perturbation of the normal stress takes the form in (54) if we apply $\Delta_{s}$ to Eq. (A16).

\section{APPENDIX B: DISPERSION RELATION}

With the solutions $g_{i}(z)$ and $g_{c}(z)$ the six remaining boundary conditions in the steady state $\omega=0[(44)-(48)$, (65)] have the following structure:

$$
\begin{aligned}
& a_{3} A_{i}+a_{4} B_{i}+a_{5} C_{i}=0, \\
& c_{3} A_{i}+c_{4} B_{i}+c_{5} C_{i}=0, \\
& d_{1} A_{c}+d_{2} B_{c}=0, \\
& e_{1} A_{c}+e_{2} B_{c}+e_{3} A_{i}+e_{4} B_{i}+e_{5} C_{i}-R \eta_{0}=0, \\
& f_{1} A_{c}+f_{2} B_{c}+f_{3} A_{i}+f_{4} B_{i}+f_{5} C_{i}=0, \\
& h_{1} A_{c}+h_{2} B_{c}+h_{3} A_{i}+h_{4} B_{i}+h_{5} C_{i}-\lambda \eta_{0}=0 .
\end{aligned}
$$

The factors multiplying the unknown coefficients $A_{c}, B_{c}, A_{i}, B_{i}, C_{i}, \eta_{0}$ are functions of $k, j, b, d, S$, and $\varepsilon$. With these six equations with six unknown coefficients we construct the determinant that has to be zero. This determinant is expressed in the following, where rows correspond to boundary equations (B1)-(B5), and (B6), respectively, and columns stand for the constants of integration $A_{c}, B_{c}, A_{i}, B_{i}, C_{i}$, and the deformation amplitude $\eta_{0}$. The elements are the coefficients multiplying each constant of integration (and deformation amplitude) in the columns in each boundary condition (row):

$$
\left[\begin{array}{cccccc}
0 & 0 & a_{3}(k, b, d) & a_{4}(k, b, d) & a_{5}(k, b, d) & 0 \\
0 & 0 & c_{3}(k, b, d) & c_{4}(k, b, d) & c_{5}(k, b, d) & 0 \\
d_{2}(k, d) & d_{2}(k, d) & 0 & 0 & 0 & 0 \\
e_{1}(k) & e_{2}(k) & e_{3}(k, b) & e_{4}(k, b) & e_{5}(k, b) & -R(j, b, S) \\
f_{1}(k, S) & f_{2}(k, S) & f_{3}(k, j, b) & f_{4}(k, j, b) & f_{5}(k, j, b) & 0 \\
h_{1}(k, j, S, \varepsilon) & h_{2}(k, j, S, \varepsilon) & h_{3}(k, j, b) & h_{4}(k, j, b) & h_{5}(k, j, b) & -\lambda
\end{array}\right],
$$

where the coefficients are

$$
\begin{aligned}
& a_{3}(k, b, d)=F_{1,2}\left(\frac{1}{2} ; \frac{1}{4}, \frac{3}{4} ; \frac{1}{4}(b-1+d)^{2} k^{2}\right), \\
& a_{4}(k, b, d)=\sqrt{b-1+d} F_{1,2}\left(\frac{3}{4} ; \frac{1}{2}, \frac{5}{4} ; \frac{1}{4}(b-1+d)^{2} k^{2}\right), \\
& a_{5}(k, b, d)=(b-1+d)^{3 / 2} F_{1,2}\left(\frac{5}{4} ; \frac{3}{2}, \frac{7}{4} ; \frac{1}{4}(b-1+d)^{2} k^{2}\right), \\
& c_{3}(k, b, d)=\frac{4}{3} k^{2} F_{1,2}\left(\frac{3}{2} ; \frac{5}{4}, \frac{7}{4} ; \frac{1}{4}(b-1+d)^{2} k^{2}\right)+\frac{16}{35} k^{4}(b-1+d)^{2} F_{1,2}\left(\frac{5}{2} ; \frac{9}{4}, \frac{11}{4} ; \frac{1}{4}(b-1+d)^{2} k^{2}\right),
\end{aligned}
$$




$$
\begin{aligned}
c_{4}(k, b, d)= & -\frac{1}{4(b-1+d)^{3 / 2}} F_{1,2}\left(\frac{3}{4} ; \frac{1}{2}, \frac{5}{4} ; \frac{1}{4}(b-1+d)^{2} k^{2}\right)+\frac{6}{5} k^{2} \sqrt{b-1+d} F_{1,2}\left(\frac{7}{4} ; \frac{3}{2}, \frac{9}{4} ; \frac{1}{4}(b-1+d)^{2} k^{2}\right) \\
& +\frac{7}{45} k^{4}(b-1+d)^{5 / 2} F_{1,2}\left(\frac{11}{4} ; \frac{5}{2}, \frac{13}{4} ; \frac{1}{4}(b-1+d)^{2} k^{2}\right), \\
c_{5}(k, b, d)= & \frac{3}{4 \sqrt{b-1+d}} F_{1,2}\left(\frac{5}{4} ; \frac{3}{2}, \frac{7}{4} ; \frac{1}{4}(b-1+d)^{2} k^{2}\right)+\frac{20}{21} k^{2}(b-1+d)^{3 / 2} F_{1,2}\left(\frac{9}{4} ; \frac{5}{2}, \frac{11}{4} ; \frac{1}{4}(b-1+d)^{2} k^{2}\right) \\
& +\frac{3}{77} k^{4}(b-1+d)^{7 / 2} F_{1,2}\left(\frac{13}{4} ; \frac{7}{2}, \frac{15}{4} ; \frac{1}{4}(b-1+d)^{2} k^{2}\right),
\end{aligned}
$$

$d_{1}(k, d)=e^{-k d}$,

$d_{2}(k, d)=e^{k d}$,

$e_{1}(k)=e_{2}(k)=-1, \quad R(j, b, S)=\sqrt{2 b j}-\frac{j}{S}$,

$e_{3}(k, b)=F_{1,2}\left(\frac{1}{2} ; \frac{1}{4}, \frac{3}{4} ; \frac{1}{4} b^{2} k^{2}\right)$,

$e_{4}(k, b)=b^{1 / 2} F_{1,2}\left(\frac{3}{4} ; \frac{1}{2}, \frac{5}{4} ; \frac{1}{4} b^{2} k^{2}\right)$,

$e_{5}(k, b)=b^{3 / 2} F_{1,2}\left(\frac{5}{4} ; \frac{3}{2}, \frac{7}{4} ; \frac{1}{4} b^{2} k^{2}\right)$,

$f_{1}(k, S)=-f_{2}(k, S)=2 k S$,

$$
\begin{aligned}
f_{3}(k, j, b)= & \sqrt{2 b j} k^{2}\left(-2 F_{1,2}\left[\frac{1}{2} ; \frac{1}{4}, \frac{3}{4} ; \frac{1}{4} b^{2} k^{2}\right]\right. \\
& \left.+4 F_{1,2}\left[\frac{3}{2} ; \frac{5}{4}, \frac{7}{4} ; \frac{1}{4} b^{2} k^{2}\right]\right) \\
& +\sqrt{2 b j} k^{2}\left(\frac{32}{35} b^{2} k^{2} F_{1,2}\left[\frac{5}{2} ; \frac{9}{4}, \frac{11}{4} ; \frac{1}{4} b^{2} k^{2}\right]\right),
\end{aligned}
$$

$$
\begin{aligned}
f_{4}(k, j, b)= & \sqrt{2 j} b k^{2}\left(-2 F_{1,2}\left(\frac{3}{4} ; \frac{1}{2}, \frac{5}{4} ; \frac{1}{4} b^{2} k^{2}\right)\right. \\
& \left.+3 F_{1,2}\left[\frac{7}{4} ; \frac{3}{2}, \frac{9}{4} ; \frac{1}{4} b^{2} k^{2}\right]\right)+\sqrt{2 j} b k^{2} \\
& \times\left(\frac{14}{45} b^{2} k^{2} F_{1,2}\left[\frac{11}{4} ; \frac{5}{2}, \frac{13}{4} ; \frac{1}{4} b^{2} k^{2}\right]\right)
\end{aligned}
$$$$
f_{5}(k, j, b)=\frac{\sqrt{2 j}}{77} 6 b^{4} k^{4} F_{1,2}\left(\frac{13}{4} ; \frac{7}{2}, \frac{15}{4} ; \frac{1}{4} b^{2} k^{2}\right)
$$$$
+\frac{\sqrt{2 j}}{77}\left(-77\left(-3+2 b^{2} k^{2}\right)\right.
$$$$
\times F_{1,2}\left(\frac{5}{4} ; \frac{3}{2}, \frac{7}{4} ; \frac{1}{4} b^{2} k^{2}\right)
$$$$
\left.+165 b^{2} k^{2} F_{1,2}\left(\frac{9}{4} ; \frac{5}{2}, \frac{11}{4} ; \frac{1}{4} b^{2} k^{2}\right)\right),
$$

$h_{1}(j, k, S, \varepsilon)=\varepsilon j k / S$,

$h_{2}(j, k, S, \varepsilon)=-\varepsilon j k / S$,

$$
\begin{aligned}
h_{3}(k, j, b)= & \sqrt{\frac{j}{2 b}}\left(-F_{1,2}\left[\frac{1}{2} ; \frac{1}{4}, \frac{3}{4} ; \frac{1}{4} b^{2} k^{2}\right]\right. \\
& \left.+\frac{8}{3} b^{2} k^{2} F_{1,2}\left[\frac{3}{2} ; \frac{5}{4}, \frac{7}{4} ; \frac{1}{4} b^{2} k^{2}\right]\right), \\
h_{4}(k, j, b)= & \frac{3 k^{2} \sqrt{2 j} b^{4}}{5} F_{1,2}\left[\frac{7}{4} ; \frac{3}{2}, \frac{9}{4} ; \frac{1}{4} b^{2} k^{2}\right], \\
h_{5}(k, j, b)= & \sqrt{2 j} b\left(F_{1,2}\left[\frac{5}{4} ; \frac{3}{2}, \frac{7}{4} ; \frac{1}{4} b^{2} k^{2}\right]\right. \\
& \left.+\frac{5}{21} b^{2} k^{2} F_{1,2}\left[\frac{9}{4} ; \frac{5}{2}, \frac{11}{4} ; \frac{1}{4} b^{2} k^{2}\right]\right), \\
\lambda=\frac{1}{U}(1+ & \left.\frac{1}{\mathrm{Bo}} k^{2}\right) .
\end{aligned}
$$

One can obtain the instability criterion by calculating the determinant, with mathematical software, putting this set of elements here supplied. Most of calculations were operated with MATHEMATICA ${ }^{17}$ where the $F_{1,2}$ appearing in the set of functions are referred to as HypergeometricPFQ. An effort is made in this work to make it easy to follow the mathematical procedure and to make possible for an eventual interested reader to make his/her own calculations and developments based on the mathematical results of this first theoretical approach to the non-ohmic/ohmic perpendicular field EHD instabilities.

${ }^{1}$ G. I. Taylor and A. D. McEwan, "The stability of a horizontal fluid interface in a vertical electric field," J. Fluid Mech. 22, 1 (1965).

${ }^{2}$ J. R. Melcher, Field-Coupled Surface Waves (MIT Press, Cambridge, 1963).

${ }^{3}$ J. R. Melcher and C. V. Smith, Jr., "Electrohydrodynamic charge relaxation and interfacial perpendicular-field instability," Phys. Fluids 12, 778 (1969).

${ }^{4}$ K. Mima, H. Ikezi, and A. Hasegawa, "Parametric excitation of collective modes in an electron layer on a liquid surface," Phys. Rev. B 14, 3953 (1976).

${ }^{5}$ R. W. Giannetta and H. Ikezi, "Nonlinear deformation of the electroncharged surface of liquid ${ }^{4} \mathrm{He}$," Phys. Rev. Lett. 47, 849 (1981).

${ }^{6} \mathrm{P}$. Atten and R. Moreau, "Stabilité électrohydrodynamique des liquides isolants soumis à une injection unipolaire," J. Mécanique 11, 471 (1972).

${ }^{7}$ D. Koulova-Nenova and P. Atten, "EHD instability of air/liquid two liquid layer system under unipolar charge injection," J. Electrost. 40-41, 179 (1997).

${ }^{8}$ P. Atten, D. Koulova-Nenova, and A. T. Pérez, “On the EHD instability of a layer of insulating liquid subjected to a unipolar charge injection due to 
corona discharge in air," Proceedings of the International Workshop on Electrical Conduction, Convection and Breakdown in Fluids, Universidad de Sevilla, 1998, pp. 47-51.

${ }^{9}$ D. Koulova-Nenova and P. Atten, "EHD convective and interfacial instabilities of conducting/insulating two liquid layer system with deformable interface," Proceeding of the International Workshop on Electrical Conduction, Convection and Breakdown in Fluids, Universidad de Sevilla, 1998, pp. 65-69.

${ }^{10} \mathrm{P}$. Atten and D. Koulova-Nenova, "On the instability between two layers of conducting and insulating liquids subjected to a DC field," Proceedings of the 13th International Conference on Dielectric Liquids (ICDL '99), Nara, Japan, 1999, pp. 277-280.

${ }^{11}$ A. T. Pérez, "Rose-window instability in low conducting liquids," J. Electrost. 40-41, 141 (1997).
${ }^{12}$ A. T. Pérez, "EHD instabilities induced by corona discharge," Proceedings of the 12th International Conference on Dielectric Liquids (ICDL '96), Roma, Italy, 1996, pp. 126-129.

${ }^{13}$ A. Castellanos, A. I. Zhakin, P. K. Watson, P. Atten, and J.-S. Chang, Electrohydrodynamics (Springer, Vienna, 1998).

${ }^{14}$ D. A. Saville, "Electrohydrodynamics: The Taylor-Melcher leaky dielectric model," Annu. Rev. Fluid Mech. 29, 27 (1997).

${ }^{15}$ S. Chandrasekhar, Hydrodynamic and Hydromagnetic Stability (Clarendon, New York, 1961).

${ }^{16} \mathrm{M}$. Abramowitz and I. Stegun, Handbook of Mathematical Functions (Dover, New York, 1965), p. 555.

${ }^{17} \mathrm{~S}$. Wolfram, The Mathematica Book, 4th ed. (Wolfram Media, Cambridge University Press, Cambridge, 1999). 